\section{Thoracic electrical bioimpedance theory and clinical possibilities in perioperative medicine}

\author{
PREDRAG STEVANOVIĆ • RADISAV ŠĆEPANOVIĆ • \\ DRAGAN RADOVANOVIĆ • ĐORĐE BAJEC • RADOSLAV PERUNOVIĆ • \\ DRAGOŠ STOJANOVIĆ • DEJAN STEVANOVIĆ
}

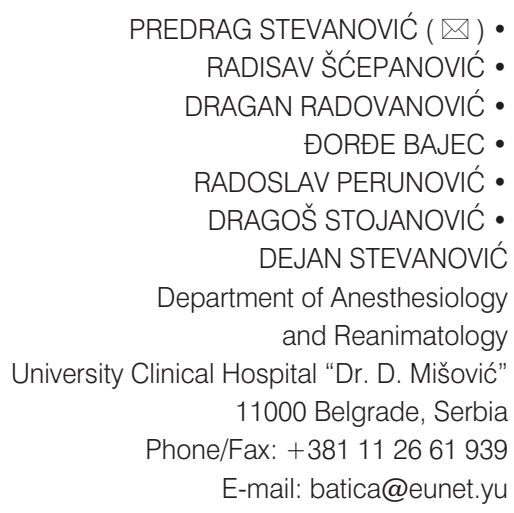

PREDRAG STEVANOVIĆ ( $\square) \bullet$ RADISAV ŠĆEPANOVIĆ • DRAGAN RADOVANOVIĆ • ĐORĐE BAJEC • RADOSLAV PERUNOVIĆ • DRAGOŠ STOJANOVIĆ • DEJAN STEVANOVIĆ

Department of Anesthesiology and Reanimatology University Clinical Hospital "Dr. D. Mišović" 11000 Belgrade, Serbia Phone/Fax: +381112661939 E-mail: batica@eunet.yu

\begin{abstract}
This article is a short review of thoracic electrical bioimpedance (TEB) theory and clinical capabilities. Cardiac output measurement is used primarily to guide therapy in complex, critically ill patients. Thoracic electrical bioimpedance is one of several noninvasive techniques that have been investigated to measure cardiac output and other hemodynamic parameters. Opinions in current literature continue to be conflicting as to the utility of thoracic electrical bioimpedance to that purpose. There is a limited number of good designed studies but they imply TEB is an accurate and reliable noninvasive method for determining cardiac output/cardiac index and it would be valuable for patients and circumstances in which intracardiac pressures and mixed venous blood samples are not necessary.
\end{abstract}

Key words: bioimpedance, non-invasive hemodynamic monitoring, cardiac output, pulmonary artery catheter, thermo dilution

There are many high-risk patients who need the measurement of cardiac output (CO) in the operation theatre and postoperative settings. $(1,2)$ It is still at present a dilemma about invasive and noninvasive way of monitoring hemodynamic function.

Invasive cardiac monitoring mea-surement is used primarily to guide therapy in complex, critically ill patients, and during the per operative period in patients with high morbidity and mortality risk. Invasive pulmonary artery catheterization has been the method of choice for the accurate evaluation of hemodynamic status.

The pulmonary artery catheter (PAC) is routinely used to measure cardiac output by thermo dilution (TD) method for patients undergoing coronary artery bypass surgery, but there are a large number of patients who undergo minor or minimally invasive surgery, who presented with severe cardiac disease. Patients in the intensive care unit who suffer from sepsis and great hemodynamic disturbance also need comprehensive cardiac monitoring. (3)

However, we can truly ask ourselves: do we really need Swan-Ganz during a laparoscopic cholecystectomy if a cardiac condition is quite bad? There are many similar situations in everyday practice.

It is widely recognized that the pulmonary artery catheter (PAC) places the patient at risk for infection and many other complications. (4) That possibility and lack of Swan-Ganz experience in a majority of anaesthetists, force a constant effort to find good, reliable and accurate noninvasive hemodynamic monitoring.
When we speak about hemodynamic monitoring, at first we think about cardiac output. There is no totally accurate method of measuring cardiac output, but it can be estimated on the basis of various assumptions. (5) CO per se means not much if we do not put it in relation with adequacy of oxygen transport, which is the ultimate goal of hemodynamic monitoring. Oxygen transport is a function of $\mathrm{CO}$ and arterial oxygen content.

Cardiac output (CO) is assessed by measuring the volume of blood pumped by the heart in one minute. The amount of blood pumped by the left ventricle in one contraction is the stroke volume. The stroke volume (SV) multiplied by the heart rate $(\mathrm{Hr})$ is the cardiac output:

$\mathrm{CO}=\mathrm{SVHr}$

$\mathrm{CO}$ is directly proportional to the mean pressure drop over the whole of the 
systemic arterial-venous circuit (i.e., mean arterial pressure (MAP) - central venous pressure (CVP)) and is inversely proportional to the total peripheral resistance (TPR) of the circuit.

$\mathrm{CO} \approx(\mathrm{MAP}-\mathrm{CVP}) / \mathrm{TPR}$

There is an analogous relationship in electricity. Ohm's Law states that flow of current ( $($, analogous to $\mathrm{CO})$ is equal to the voltage drop $(\mathrm{V}$, analogous to the pressure drop) between two ends of a circuit, divided by the resistance to current flow ( $R$, analogous to TPR). For direct current we can state:

$\mathrm{I}=\mathrm{V} / \mathrm{R}$

$\mathrm{R}=\mathrm{V} / \mathrm{I}$

If $\mathrm{I}=$ constant, a change in resistance $(\triangle R)$ is proportional to a change in voltage $(\Delta V)$ :

$\Delta R \approx \Delta V$

For alternating current flow, resistance is known as impedance (Z) and is complex, frequency-dependent parameter. For alternating current we can say that:

$\mathrm{Z}=\mathrm{V} / \mathrm{I}$

$Z$ is measured in ohms. If I remains constant, then periodic changes in voltage $(\Delta \mathrm{V})$ produce concurrent changes in impedance $(\Delta Z)$ :

$\Delta Z \approx \Delta V$

This simple equation is fundamental to understanding SV determination by thoracic electrical bioimpedance (TEB). Thoracic Electrical Bioimpedance (TEB), with a symbol Z, is an electrical resistance of the thorax to a high-frequency, very-low magnitude TEB measurement current. TEB utilizes a patient's thorax as an impedance transducer.

The impedance to alternating current flow of a simple cylindrical electrical conductor is equal to its specific resistance $(\rho)$ times it length $(L)$, divided by its cross-sectional area $(A)$ :

$Z=\rho L / A)$
For simplicity, the thorax can be modeled as a cylindrical conductor of length $\mathrm{L}$, which has embedded, in parallel with the thoracic length, a smaller cylindrical conductor, representing the great vessels (figure 1). In that small cylinder representing the vessels is blood of specific resistance $\rho_{b}$, with cross-sec-

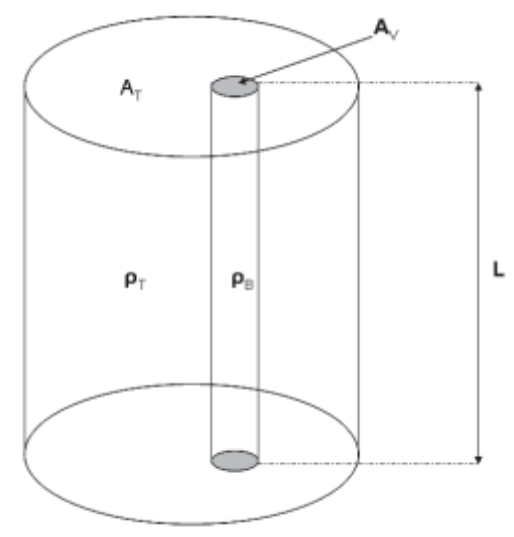

Figure 1. Simple model of human thorax. Small cylinder represents great vessels with cross-sectional area $A v$, length $L$ and specific resistance of blood $\rho b$. Remaining thoracic volume has cross-sectional area AT, length $L$ and specific resistance $\rho T$ ( $\rho b<<\rho T)$.

tional area labeled $A_{V}$. The remaining volume of the cylinder is assumed to be homogenous with specific resistance $\rho_{T}$ ( $\rho$ thorax) and cross-sectional area labeled $\mathrm{A}_{\mathrm{T}}$.

It is assumed that the small cylinder has a varying cylindrical cross-sectional area: $A_{V}+\Delta A_{V}(t)$, caused by aortic and pulmonary artery pulsations with each heart beat. If we assumed that $\rho_{\mathrm{b}}<<\rho_{\mathrm{T}}$ we can conclude that the change in $Z$ with each heart beat is caused mostly by changes in the crosssectional areas of the great vessels $\triangle A_{V}(t)$. Synthesizing this theory with Ohm's Law, it comes about that the periodic increases in cross-sectional area ( $L$ remaining constant) and volume of the great vessels $\left(\triangle A_{V}\right)$ must cause corresponding decreases in thoracic impedance and vice versa.

Systemic aortic blood pressure and expansion of the great vessels are accompanied by simultaneous chan- ges in thoracic impedance $(\Delta Z)$. The increased thoracic conductivity is caused by the systolic pumping of blood into the great vessels from the ventricles, caused by ventricular systole, and it is registered as decreases in impedance to current flow.

Time-varying pulsatile changes in aortic and pulmonic blood volume $(\Delta \mathrm{V})$ are directly proportional to the time-varying, cardiac-induced impedance change $(\triangle Z)$ :

$\Delta V(t) \approx \rho_{b}\left(L^{2} / Z_{0}{ }^{2}\right) \Delta Z ;$ where $Z_{0}$ is the nonpulsatile base impedance measured.

The Base Impedance $\left(Z_{0}\right)$ is indirectly proportional to total content of thoracic fluids. We cannot identify individual conductance contributions of the intravascular, intra-alveolar and interstitial compartments and therefore, we can see the thoracic fluid conductivity, which is then directly proportional to the thoracic fluids content (TFC). The TEB variations and changes $(\triangle Z)$ are produced by:

- slow changes of fluid levels in all thoracic compartments,

- tidal changes of venous and pulmonary blood volume caused by respiration,

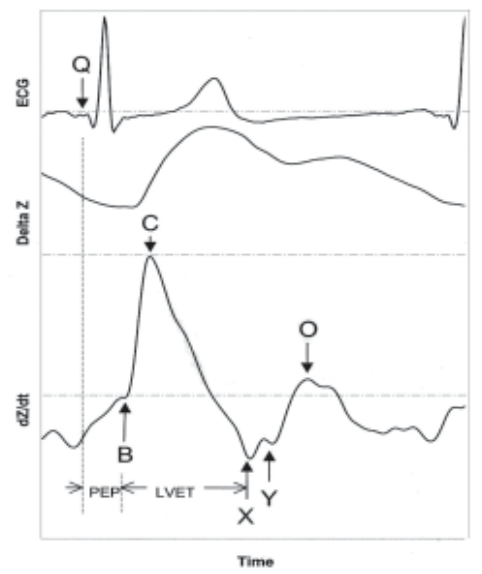

Figure 2. ECG and ICG Waveforms. $\mathbf{Q}=$ Ventricular depolarization, $\mathbf{B}=$ Opening aortic and pulmonic valves, $C=$ Maximal slope $\Delta Z, X=$ Closure aortic valve, $Y=$ closure of pulmonic valve, $\mathbf{O}=$ Opening mitral valve / rapid filling of ventricles. 
- volumetric and velocity (alignment of planes of erythrocytes as a function of blood velocity) changes of aortic blood produced by the heart's pumping activity.

The cardiac origin of $\Delta Z$ is obvious when viewed in light of its electrocardiogram (ECG) counterpart (figure 2). Like ECG, TEB has important waveform characteristics that reflect significant points in the cardiac cycle (figure 3 ). The change in impedance $(\Delta Z)$ is measured from the baseline impedance $Z_{0}$ (shown as dotted line). Baseline impedance is inversely proportional to the amount of conductive material in the thorax. The thoracic fluid content parameter (TFC) is the inverse of baseline impedance, so TFC is directly proportional to the amount of conductive material in the thorax. As fluid in the chest increases,

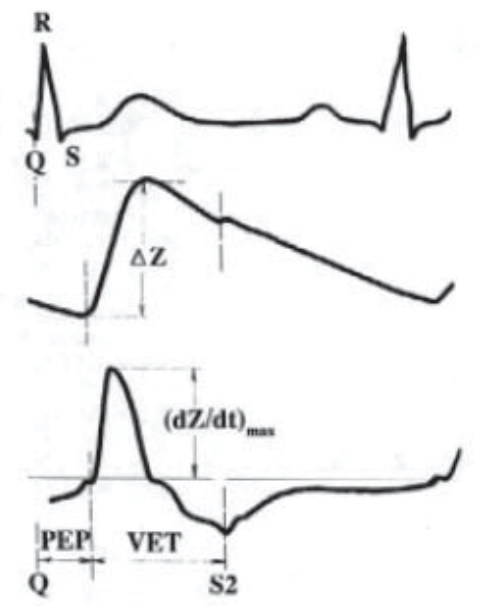

Figure 3. The timing relationship between ECG and thoracic electrical bioimpedance:myocardialcontraction starts at the Q-time of the ECG QRS complex. The Pre-Ejection Period (PEP) (isovolumic contraction) is defined as the elapsed time between the Q-time of the QRS complex and the opening of aortic valve. The ejection phase, outlined by the Ventricular Ejection Time (VET), starts by opening of aortic valve and ends by its closure (S2-time). During the initial portion of ejection phase the aorta distends and the thorax, therefore, becomes more conductive; at the same time the velocity of blood increases, more erythrocytes are aligned so their planes are parallel with the main axis of aorta and, therefore, the blood becomes more conductive.
TFC increases. The first derivative (dz/ $\mathrm{dt}$ ) of the delta $Z$ waveform is used to identify the maximum upslope, shown as point $\mathrm{C}$. This is used to calculate Velocity Index $(\mathrm{VI})$, which is indicative of aortic blood velocity. As the heart pumping ability is impaired, $\mathrm{VI}$ decreases. The left ventricular ejection time (LVET) is the time from the opening of the aortic valve (B point) to the closing (X point). As the heart loses its ability to contract (systolic function worsens), LVET shortens.

The patient is connected to the monitor via a patient cable attached to eight solid-gel, disposable electrodes (figure 4). The TEB measurement current is passed through the thorax in a direction parallel with the spine between the beginning of the thorax (the line at the root of the neck) and the end of the thorax (at the level of diaphragm - the xiphoid process level). Four dual sensors with eight lead wires are placed on neck and chest. Current transmitted by outer electrodes seek the path of least resistance: blood filled aorta. Baseline impedance (resistance) is measured using inner electrodes. With each heartbeat, blood volume and velocity in the aorta changes. Corresponding change in impedance is measured. Baseline and changes in impedance are used to measure and calculate hemodynamic parameters.

These four electrodes also detect four different vectors of the ECG signal. The Heart Rate (HR) is derived from the $R-R$ intervals of the ECG signal. Due to the anatomical shape of the thorax, a preferential placement for all eight electrodes is along the frontal plane - the widest thoracic dimension.

With TEB we can get many noninvasive parameters (table1).

From a clinical point of view, we can divide all this parameters into four groups (table 2).

We can use TEB for hemodynamic evaluation in many circumstances:

- Fluid management for heart failure,

- Differentiation of cardiogenic from pulmonary causes of acute dyspnea,

- Optimization of atrioventricular interval in cardiac pacemakers,
- Monitoring of patients for early diagnosis of rejection after heart transplantation,

- Management of drug-resistant hypertension,

- Evaluation of hemodynamic response in dehydrated patients,

- Management of patients with severe cardiac illness during surgery,

- Management of patients during intensive care,

- Management of patients in the Emergency Department,

- Noninvasive monitoring for early recognition and treatment of shock in highrisk trauma and surgical patients.

In the per operative fluid management, which is everyday practice, thoracic fluid content (TFC) could be very applicable parameter.

- A single TFC measurement indicates total conductivity of the chest that is affected by both intravascular and extra vascular fluid.

- Changes in TFC from a previous measurement represent changes in total fluid of the chest, intravascular and/or extra vascular volume.

Although TFC does not correlate with PCWP, it is a very good indicator of thoracic fluid. Changes in TFC are very reliable indicators of changes in intraor extra-vascular fluid volume.

There are many decompensated heart failure patients preoperative and in the intensive care unit. It is impossible to imagine that all of them are candidates for invasive monitoring. There is a place for TEB in the differentiation of cardiogenic from pulmonary causes of acute dyspnea when history, physical examination, and standard assessment tools provide insufficient information. Some authors investigate usefulness of the impedance value on a scale of 1 to 10 for each heart failure patient. (6) The mean usefulness rating was 7.9 (95\% Confidence Interval: 6.5 - 9.3). The researchers concluded that the baseline impedance value obtained from noninvasive hemodynamic monitoring with TEB accurately predicted the presence and severity of pulmonary 
a)

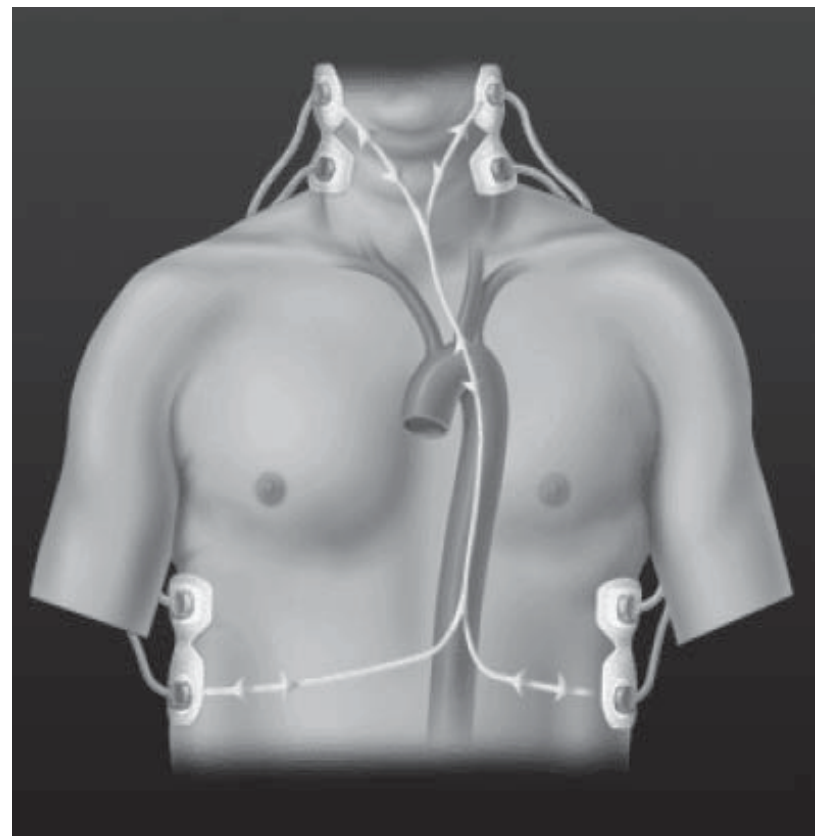

b)

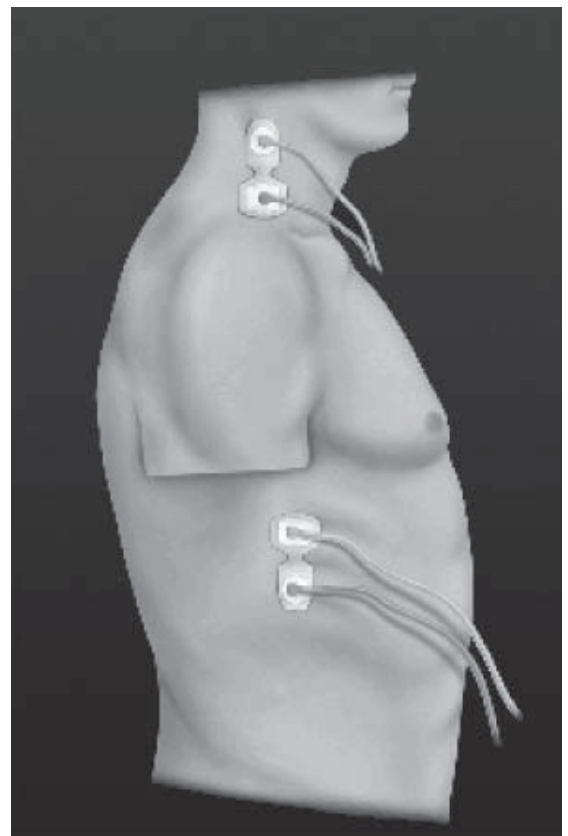

Figure 4. Location of the 8 electrodes along the TEB transducer - a patient's thorax. The top and bottom pair is a source and sink of the TEB measurement current, the inner pairs, located at the root of the neck (the beginning of the transducer) and the diaphragm level, i.e., the xiphoid process level (the end of the transducer), are used for sensing both the TEB signal and 4 different vectors of the ECG signal. a) Front view b) Lateral view

edema and can serve as an important adjunct in the treatment of heart failure patients.

In many operative procedures TEB may be used instead of invasive techniques as it is accurate, reliable and simple to use, with proven efficacy. (7) It has the additional advantage of allowing continuous monitoring of cardiac index by noninvasive means, and gives the anesthetist information regarding stroke volume, cardiac and ejection velocity indices in a "real-time" manner, in response to drugs or surgical stimuli.

It is well known that respiration and artificial ventilation result in cyclic fluctuations of the cardiac output determination by thermodilution (TD). Because of the modulation shift during changing ventilation patterns, i.e. different levels of PEEP, researchers doubt that the TD technique is an appropriate method for studying modulations of cardiac output dependent on artificial ventilation.

Castor and colleges in their study find that TEB is an accurate and reproducible method for determination of cardiac output under these changing respiratory patterns during artificial ventilation. (8)
There are many studies that compare TEB and direct Fick cardiac output methods. In a prospective study, Van DeWater et al. (9) compared the accuracy of TEB to thermodilution (CO-TD) in postoperative coronary artery bypass graft (CABG) patients. This study took place in a cardiovascular-thoracic surgery intensive care unit (ICU). The study included 53 post-CABG patients from whom 210 pairs of cardiac output measurements were made. The TEB cardiac output was determined simultaneously with the TD cardiac output. The authors reported that when comparing TEB to TD, they found that the bias, precision, correlation slope, and intercept were equivalent to TD. The authors stated that in those circumstances in which intracardiac pressures and mixed venous blood samples are not necessary, TEB is preferable to invasive TD method in determining cardiac output. The authors stated that the TEB monitor allows for quick and easy cardiac output monitoring and systemic vascular resistance in clinical areas where the pulmonary artery catheter is not typically utilized (e.g., emergency department, sub acute care, hypertension and heart failure). "The latest TEB technology for determining $\mathrm{CO}$ is less variable and more reproducible in an intrapatient sense than is COTD." TEB is reproducible, especially in comparison to serial measurements using Thermo dilution. The historical standard for hemodynamic measurement, thermo dilution (TD), shows only modest correlation when compared to itself. The SD for thermodilution is about 1 liter/minute or about $20 \%$ of the average CO. TEB shows very high correlation when compared to itself, and lower standard deviation for multiple TEB measurements.

In the other study of 23 adults in the intraoperative and post operative settings, the correlation coefficient between the two methods was $r=0.89, p<0.001$. (10) In a study of 68 critically ill patients, changes in cardiac output estimated by TEB were found to closely correlate with values obtained with TD method $r$ $=0.86, p<0.001$. (11)

Shock and shock-related organ failure account for most deaths in trauma and surgical patients. The study of Asensio 
and colleges examines the use of noninvasive methods, including thoracic electrical bioimpedance (TEB) for early recognition and treatment of shock in high-risk trauma and surgical patients. (12) This study involved a series of trauma patients entering the emergency department. It reviews the use of invasive and noninvasive monitoring methods in these patients to describe the temporal patterns of cardiac, pulmonary, and tissue perfusion functions in the early phase of trauma. The authors concluded that noninvasive monitoring is easy to apply, safe, inexpensive, and more cost-effective than invasive monitoring. High-risk patients might profit by earlier noninvasive hemodynamic monitoring. If noninvasive monitoring identifies circulatory problems earlier, then even more expeditious therapy could be given to achieve optimal physiologic goals that improve outcome.

Shoemaker and colleges in their study evaluate the feasibility of multicomponent hemodynamic monitoring in critical emergency patients and compares the technique of TEB with simultaneous monitoring by the pulmonary artery thermodilution catheter. (13) The researchers concluded that: "Noninvasive monitoring can provide hemodynamic and perfusion information previously available by invasive thermodilution catheters. Such noninvasive monitoring can display continuous on-line real-time data, allowing immediate recognition of circulatory abnormalities and providing a means to titrate therapy to appropriate therapeutic goals."

Potential limitations in use of TEB are states with HR > 250 bpm, septic shock (end stage sepsis), severe aortic valve regurgitation, extremely high blood pressure (MAP > 130) and intraaortic balloon pump. Also, extremely tall $(>210 \mathrm{~cm})$ and low $(<120 \mathrm{~cm})$ patients and patients under $35 \mathrm{~kg}$ and over 170 $\mathrm{kg}$ lose out the accuracy of measurement by TEB.

\section{Conclusion}

Thoracic electrical bioimpedance is one of several noninvasive techniques that have been used to measure cardiac

Table 1. Parameters captured by TEB measurement.

\begin{tabular}{|c|c|}
\hline Parameter & Range (Normal Values) \\
\hline Cardiac Output & $\begin{array}{l}\text { 0-30 L/min } \\
\text { (Variable Normal Values) }\end{array}$ \\
\hline Cardiac index & $\begin{array}{l}0-15 \mathrm{~L} / \mathrm{min} / \mathrm{m}^{2} \\
\left(2.5-4.7 \mathrm{~L} / \mathrm{min} / \mathrm{m}^{2}\right)\end{array}$ \\
\hline Stroke Volume & $\begin{array}{l}\text { 0-250 ml } \\
\text { (Variable Normal Values) }\end{array}$ \\
\hline Stroke Index & $\begin{array}{l}0-125 \mathrm{ml} / \mathrm{m}^{2} \\
\left(35-65 \mathrm{ml} / \mathrm{m}^{2}\right)\end{array}$ \\
\hline Systemic Vascular Resistance & $\begin{array}{l}\text { 0-5000 dyne-sec-cm-5 } \\
(742-1378 \text { dyne-sec-cm-5) }\end{array}$ \\
\hline $\begin{array}{l}\text { Systemic Vascular Resistance } \\
\text { Index }\end{array}$ & $\begin{array}{l}0-10,000 \text { dyne-sec-cm }{ }^{-5}-m^{2} \\
(1337-2483 \text { dyne-sec-cm-5-m²) }\end{array}$ \\
\hline Thoracic Fluid Content & $\begin{array}{l}10-150 / \mathrm{k} \Omega \\
(30-50 / \mathrm{k} \Omega \text { - males } \\
21-37 / \mathrm{k} \Omega \text { - females) }\end{array}$ \\
\hline Pre-ejection Period & $\begin{array}{l}\text { 0-1000 msec } \\
\text { (Variable Normal Values) }\end{array}$ \\
\hline Acceleration Index & $\begin{array}{l}1-400 / 100 s^{2} \\
\left(70-150 / 100 s^{2}-\text { males }\right. \\
\left.90-170 / 100 s^{2}-\text { females }\right)\end{array}$ \\
\hline LV Ejection Time & $\begin{array}{l}0-1500 \text { msec } \\
\text { (Variable Normal Values) }\end{array}$ \\
\hline Velocity Index & $\begin{array}{l}0-200 / 1000 s \\
(33-65 / 1000 s)\end{array}$ \\
\hline Systolic Time Ratio & $\begin{array}{l}0-1.0 \\
(0.3-0.5)\end{array}$ \\
\hline Left Stroke Work Index & $\begin{array}{l}0-200 \mathrm{gm}-\mathrm{m} / \mathrm{m}^{2} \\
\left(51.6-74.3 \mathrm{gm}-\mathrm{m} / \mathrm{m}^{2}\right)\end{array}$ \\
\hline Indexed Left Cardiac Work & $\begin{array}{l}0-25 \mathrm{~kg}-\mathrm{m} / \mathrm{m}^{2} \\
\left(3.0-5.5 \mathrm{~kg}-\mathrm{m} / \mathrm{m}^{2}\right)\end{array}$ \\
\hline Heart Rate & $\begin{array}{l}40-250 \text { beats } / \mathrm{min} \\
(58-86 \text { beats/min) }\end{array}$ \\
\hline Estimated Delivered $\mathrm{O}_{2}$ Index & $\begin{array}{l}0-2000 \mathrm{ml} / \mathrm{min} / \mathrm{m}^{2} \\
\left(550-650 \mathrm{ml} / \mathrm{min} / \mathrm{m}^{2}\right)\end{array}$ \\
\hline
\end{tabular}

output and other hemodynamic parameters. It is a simple, cost-effective tool for clinical assessment. TEB repeatedly demonstrated good correlation with other clinical measures of cardiac function and associated parameters. The advancements in hardware, enhanced algorithms used in software, and clinical experience may well expand the clinical applications for this continuous noninvasive hemodynamic monitoring. We must say that evidence in the current literature continue to be conflicting as to the utility of TEB in measuring hemodynamic parameters but TEB has demonstrated clinically acceptable accuracy with lower cost per patient in those circumstances in which intracardiac pressures and mixed venous blood samples are not necessary. Furthermore, it enables us to quickly and easily monitor CO and SVR in clinical areas where the invasive monitoring is not typically utilized. The use of TEB may potentially improve patient outcomes. 
Table 2. The hemodynamic parameters measured and calculated by TEB reflect cardiac flow (output) and the four determinants of cardiac output (HR, preload, afterload, and contractility).

\begin{tabular}{ll}
\hline Flow & Stroke Volume / Index (SV / SI) \\
& Cardiac Output / Index (CO / Cl) \\
\hline \multirow{2}{*}{ Resistance } & Systemic Vascular Resistance (SVR) \\
& Index (SVRI) \\
\hline \multirow{3}{*}{ Contractility } & Systolic Time Ratio (STR) \\
& Pre-ejection Period (PEP) \\
& LV Ejection Time (LVET) \\
& Velocity Index (VI) \\
\hline Fluid & Acceleration Index (ACI) \\
\hline
\end{tabular}

\section{REFERENCES}

1. Connors AF Jr, Speroff T, Dawson NV, Thomas C, Harrell FE Jr, Wagner D, et al, editors. The effectiveness of right heart catheterization in the initial care of critically ill patients. JAMA 1996;276:889-97.

2. Van De Water JM, Miller TW, Vogel RL, Mount BE, Dalton ML. Impedance cardiography: the next vital sign technology? Chest 2003;123:2028-33.

3. Rivers E, Nguyen B, Havstad S, Ressler J, Muzzin A, Knoblich B, et al, editors. Early goal-directed therapy in the treatment of severe sepsis and septic shock. N Engl J Med 2001;345:1368-77.

4. Sandham JD, Hull RD, Grant RF. A randomized controlled trial of the use of pulmonary artery catheters in high-risk surgical patients. N Engl J Med 2003;348:5-14.

5. Shoemaker WC, Appel PL, Kram HB, Nathan RC, Thompson JL. Multicomponent noninvasive physiologic monitoring of circulatory function. Crit Care Med 1988;16:482-90.

6. Milzman PD, Hogan C, Zlindenny A, Battiata A, Janchar T. The utility of thoracic impedance to evaluate chest radiograph changes from acute heart failure patients in the emergency department. 1998;4(3):37.

7. Koksoy C, Kuzu MA, Kurt I, Kurt N, Yerdel MA, Tezcan C, et al, editors. Hemodynamic effects of pneumoperitoneum during laparoscopic cholecystectomy: a prospective comparative study using bioimpedance cardiography. Br J Surg 1995;82:972-4.

8. Castor G, Molter G, Helms J, Niedermark I, Altmayer P. Determination of cardiac output during positive end-expiratory pressure-noninvasive electrical bioimpedance compared with standard thermodilution. Crit Care Med 1990;5:544-6.

9. Van De Water MJ, Miller WT, Vogel LR, Mount EB, Dalton LM. Impedance cardiography: the next vital sign technology? Chest 2003;123:2028-33.

10. Thangathurai D, Charbonnet C, Roessler P, Wo CC, Mikhail M, Yoahida R, et al, editors. Continuous intraoperative noninvasive cardiac output monitoring using a new thoracic bioimpedance device. J Cardiothorac Vasc Anesth 1997;11:440-4.

11. Shoemaker WC, Wo CC, Bishop MH, Appel PL, Van de Water JM, Harrington GR, et al, editors. Multicenter trial of a new thoracic electrical Bioimpedance device for cardiac output estimation. Crit Care Med 1994;22(12):1907-12.

12. Asensio J, Demetriades D, Berne T, Shoemaker W. Invasive and noninvasive monitoring for early recognition and treatment of shock in high-risk trauma and surgical patients. Surg Clin North Am 1996;76(4):985-97.

13. Shoemaker WC, Wo CC, Bishop MH, Thangathurai D, Patil RS. Noninvasive hemodynamic monitoring of critical patients in the emergency department. Acad Emerg Med 1996;3:675-81. 